\title{
Hydraulic transport regimes of grains in a millifluidic confinement
}

\author{
Guillaume Dumazer ${ }^{1, *}$ and Étienne Gagnepain ${ }^{1}$ \\ ${ }^{1}$ Mines Saint-Étienne, Univ. Lyon, CNRS, UMR 5307 LGF, Center SPIN, F-42023 Saint-Étienne, France
}

\begin{abstract}
Granular matter transported by hydraulic conveying behaves under several transport regimes. The techncal challenge is to predict the dilute or dense regime of transport as well as the pressure drop required in order to design properly a granular hydraulic conveying system. We address this problem with a laboratory scale experimental setup able to reproduce the key features of hydraulic conveying of grains. Pressure drop measurements as well as image analysis have been used to characterize a slug transport regime and its crossover towards a moving bed transport regime. A dimensionless characteristic curves diagram has been established and a reasonably good pressure drop prediction with a generic power law is explained in details. This preliminary work has the potential to open perspectives for the design of a predictive tool for pressure drops in hydraulic conveying systems.
\end{abstract}

\section{Introduction}

Transporting granular material is essential for a large range of industrial activities working from granulated raw material. From powder handling in selective laser sintering additive manufacturing [1], factory processes requiring continuous supply with a powder, to the extraction of drill cuttings from a well [2], conveying grains techniques cover very different scales. Fluidization systems such as pneumatic or hydraulic conveying represent scientifically challenging problems due to the complex interaction between the suspending fluid phase and the granular phase [3]. A large diversity of particle conveying regimes have been reported in the literature [4-6]. Authors distinguish dilute regimes from dense regimes. The concentration of solid particles matters but not only as dilute regime are also defined with rather homogeneous concentration of solid particles in the flow. Dilute regimes are then by definition close to granular suspensions [7]. Dense regimes have technological advantages over the dilute ones in terms of efficiency, conveying duct erosion or limited solid particles fragmentation. However their behaviour is complex as the solid particles are packed and interact by solid friction with other neighbouring particles as well as with solid walls. Typical dense regimes feature the transport of particles in slug flow or plug flow regime, where granular matter fills the entire cross section of the conveying duct over a finite length and gets pushed by the viscous flow [6]. One must also cite here the moving bed regime in horizontal conveying geometries when the viscous flow drags the sedimented bed of grains lying on the bottom solid wall [4].

We decided here to investigate the case of the hydraulic transport of a glass beads in a horizontal millifluidic tube. From this idealized system we characterized experimen-

*e-mail: guillaume.dumazer@emse.fr A video is available at https://doi.org/10.48448/72bw-a052

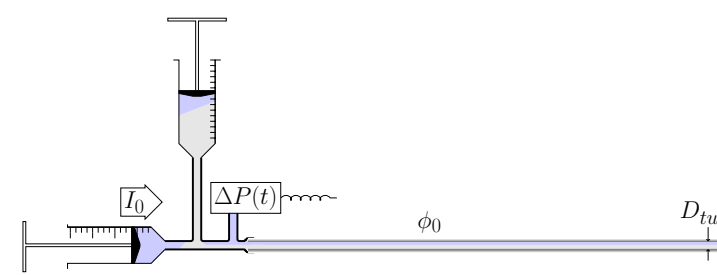

(a)

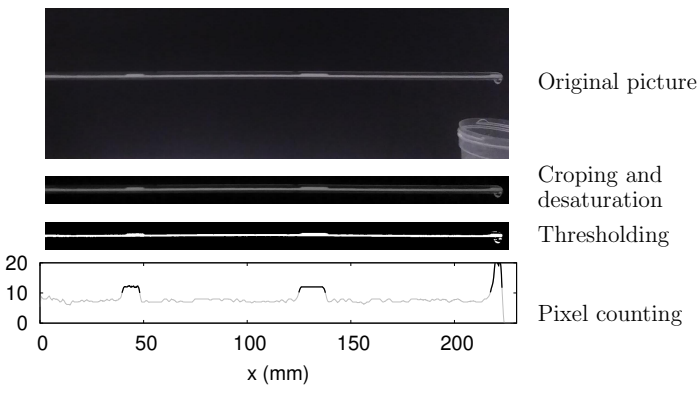

(b)

Figure 1. (a) Schematics of the experimental setup. (b) Steps illustrating the image treatment procedure from original image to granular matter profile.

tally the crossover from a dense slug flow regime to a moving bed regime. We introduced a dimensionless specific curve diagram where both regimes follow a unique trend.

\section{Material and Methods}

The experimental setup is illustrated on Fig. 1(a). We use an horizontal glass tube of internal diameter $2 \mathrm{~mm}$. A syringe pump imposes a fixed flow rate $I_{0}$ of filtered water in the tube, whose outlet is open to the atmosphere. A second syringe filled with glass beads of diameter $100 \mu \mathrm{m}$ 


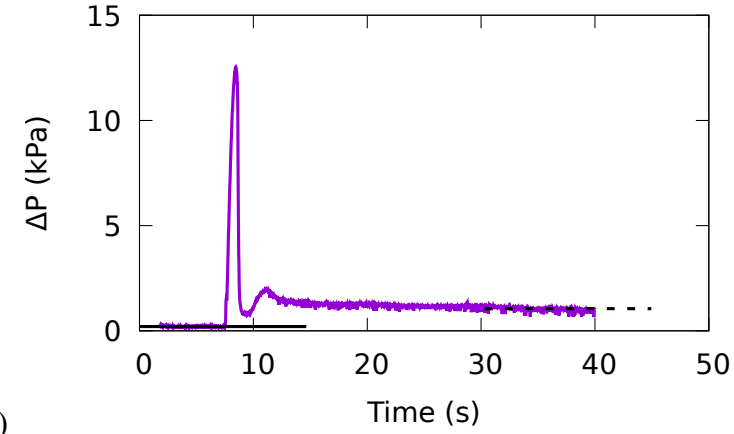

(a)

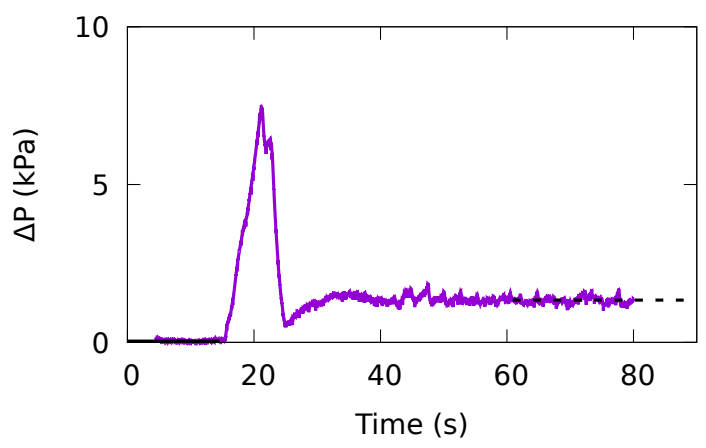

(b)

Figure 2. Pressure time series measured with an imposed flow rate (a) $I_{0}=10 \mathrm{~mL} \cdot \mathrm{min}^{-1}$ and (b) $I_{0}=2 \mathrm{~mL} \cdot \mathrm{min}^{-1}$. Solid and dashed lines mark the steady pressure plateaus obtained without any grains.

acting as a granular matter reservoir is discharging into the stream at the tube's inlet. A differential pressure sensor measures the pressure drop $\Delta P$ between the tube's inlet and the atmosphere. The flow inside the tube is recorded by a camera with frame rate $24 \mathrm{fps}$.

The experiments begin by setting up a steady flow of water only. The granular matter is then poured into the stream of water before the tube's inlet. To accelerate the relaxation towards a steady hydraulic transport of grains, a quick granular flow is applied by pressing the syringe storing the granular matter. By doing so a front of fluidized grains spreads all along the tube and it results in a homogeneous sedimented layer of grains. This method to shorten the potentially long transient regime of granular matter spread in the tube explains the pressure peak visible between the two starting and ending plateaus, corresponding respectively to the pressure drop due to the Hagen-Poiseuille flow of water only, and to the pressure drop due to the hydraulic transport of granular matter, see Fig. 2.

The experiment ends when the syringe pump is empty. Several values of flow rate $I_{0} \in[1-20] \mathrm{mL} \cdot \mathrm{min}^{-1}$ have been tested. The reproducibility of the observations have been ensured by repeating each experiments three times.

The present setup do not allow the user to impose a fixed mass rate of grains, since the vertical syringe storing the granular matter can either be open or close. The mass rate $W$ of transported granular matter is then measured by collecting the mixture of water and grains at the tube's outlet over a controlled duration $\Delta t$. The collected mixture mass $\Delta m_{\text {tot }}$ can be decomposed into two contributions :

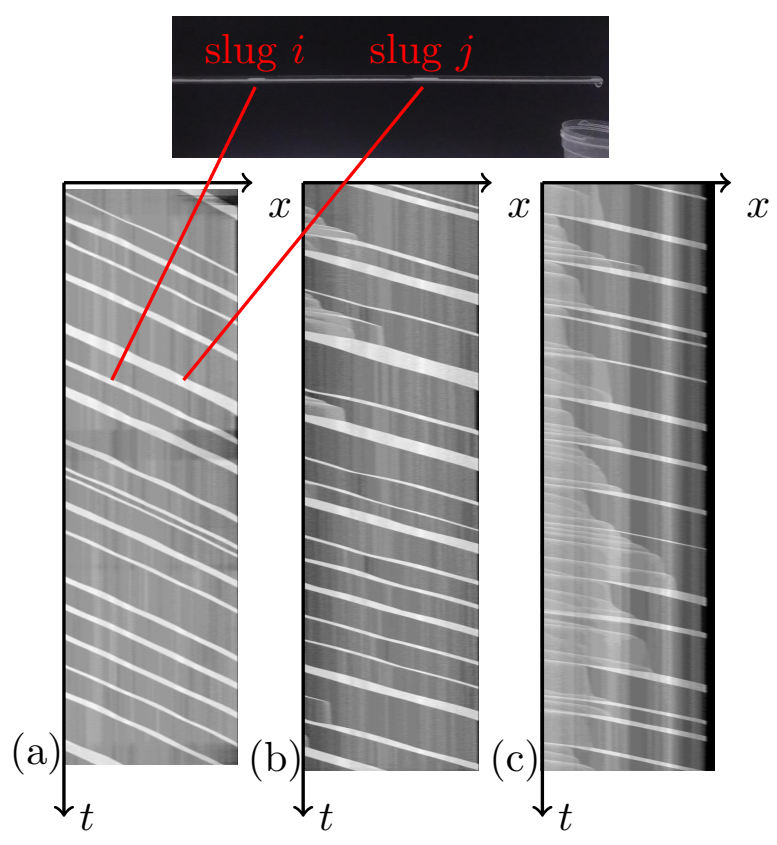

Figure 3. Spatio-temporal diagrams obtained from a section of the tube of length $234 \mathrm{~mm}$ located left of the tube's outlet so that the imposed flow rates (a) $I_{0}=1 \mathrm{~mL} \cdot \mathrm{min}^{-1}$, (b) $I_{0}=2 \mathrm{~mL} \cdot \mathrm{min}^{-1}$, and (c) $I_{0}=3 \mathrm{~mL} \cdot \mathrm{min}^{-1}$, are oriented rightwards. Time develops downwards for $231 \mathrm{~s}$.

(i) the mass of water $\Delta m_{w}=I_{0} \Delta t$; (ii) the mass of transported grains $\Delta m_{g}=W \Delta t$. So the mass rate is calculated as $W=\left(\Delta m_{\text {tot }}-I_{0} \Delta t\right) / \Delta t$.

The images recorded are treated after black and white conversion and thresholding to isolate granular material only, see Fig. 1(b). The profile of granular material along the tube is obtained from the thresholded image by counting the number of white pixels at each $x$-coordinate values, the $x$-direction being the horizontal longitudinal tube's direction. The temporal dynamics is represented by means of a spatio-temporal diagram, see Fig. 3. All granular profiles along the tube obtained after image treatment are stacked on top of each other from top to bottom with time. This way the ordinate corresponds to the temporal axis developing downwards, and the abscissae are the longitudinal coordinates. The local amount of grains at a position $x$ is converted in grey scale where light values correspond to large amounts of grains, whereas dark values correspond to small quantities of grains. In the examples on the Fig. 3 travelling slugs can be seen as inclined light stripes, whose slopes correspond to their speeds and directions. In between these light stripes the intermediate grey level background corresponds to the sedimented bed resting on the bottom side of the tube.

\section{Results}

At flow rates $I_{0} \in[5-20] \mathrm{mL} \cdot \mathrm{min}^{-1}$ we observe a regime where the granular matter is transported homogeneously as a moving sedimented bed. The granular bed creeps 
along the bottom side of the tube. The pressure drop measured during the hydraulic transport of particles exhibits a clear increase of approximately one order of magnitude compared to the pure liquid flow, see Fig. 2(a).

For smaller flow rates $I_{0} \in[1-4] \mathrm{mL} \cdot \mathrm{min}^{-1}$ the transport of granular matter crosses over towards a regime of slugs formation. A static granular bed with no clear grains movement covers the bottom side of the tube. At the tube's inlet a continuous feed of grains forms local accumulations of grains. One travelling slug germinates once the grains accumulation fills the entire cross section of the tube. Slugs move by accumulating granular matter at its front and rejecting matter at its back. The slug displacement is driven by the imposed flow rate $I_{0}$. On the spatiotemporal diagrams in Fig. 3, the slopes of the white stripes indicate the travelling speeds of the slugs for flow rates $I_{0} \in[1-3] \mathrm{mL} \cdot \mathrm{min}^{-1}$. As the flow rate increases the slopes flatten, this corresponds to an increase in travelling speeds. A viscous drag acts on the porous media formed by the grains accumulated in the slug. This viscous resistivity explains how the imposed flow rate pushes forward the slugs. However the relationship between imposed flow rate and slugs speeds doesn't seem to increase linearly. The pressure drop in this regime exhibits a large increase compared to the Hagen-Poiseuille prediction with pure liquid, see Fig. 2(b). The pressure plateau at the end of the transient setting of travelling slugs regime exhibits fluctuations. The intermittent dynamics of slugs germination, travelling through the tube and exit at outlet seems to be reflected in the pressure time-series.

\section{Discussion}

\subsection{Transition}

The pressure drops obtained for hydraulic transport of grains for different imposed flow rate values $I_{0}$ has been plotted on Fig. 4. We observe that the two hydraulic transport regimes exhibit opposite evolutions of the pressure drop with the imposed flow rate $I_{0}$. In the hydraulic regimes featuring the formation and travelling of granular slugs, i. e. for $I_{0} \leq 4 \mathrm{~mL} \cdot \mathrm{min}^{-1}$, the pressure drop increases with the imposed flow rate. On the other hand, for hydraulic regimes where the entire sedimented bed is put in motion and moves with the flow, i. e. for $I_{0} \geq 5$ $\mathrm{mL} \cdot \mathrm{min}^{-1}$, the pressure drop decreases with the flow rate. A maximum is observed for flow rate $I_{0} \approx 4 \mathrm{~mL} \cdot \mathrm{min}^{-1}$ where the hydraulic transport regimes is seen to crossover. The Hagen-Poiseuille prediction gives a lower bound for $\Delta P$. The pressure drop is therefore expected to exceed the local maximum obtained for $I_{0} \approx 4 \mathrm{~mL} \cdot \mathrm{min}^{-1}$ for very large imposed flow rate values.

\subsection{Dimensionless characteristic curves}

It is common to characterize the conveying of grains by a carrying fluid with the so-called specific curves in a diagram showing the pressure drop $\Delta P / L$ in a conveying line of length $L$ and radius $R$ versus the carrying fluid velocity

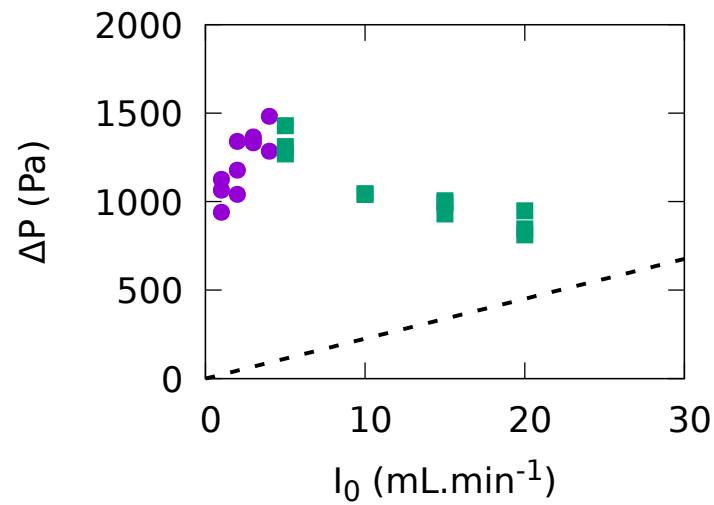

Figure 4. Pressure drop $\Delta P$ measured after granular matter is introduced in the stream of water imposed by the syringe pump at flow rate $I_{0}$. Rounded symbols correspond to hydraulic transport regimes exhibiting slugs formation and travelling, squared symbols correspond to hydraulic transport exhibiting moving bed characteristics. The dashed line indicates the Hagen-Poiseuille pressure drop prediction for pure water.

$v_{G}=I_{0} /\left(\pi R^{2}\right)$, see Fig. 5. One limiting curve in the diagram corresponds to the pressure drop evolution of a pure carrying fluid, given by a Hagen-Poiseuille law :

$$
\left.\frac{\Delta P}{L}\right|_{0}=\frac{8 \eta_{0} v_{G}}{R^{2}},
$$

for a carrying fluid of viscosity $\eta_{0}$. A second limiting curve corresponds to the pressure drop due to the carrying fluid flowing through the tube entirely filled and obstructed with packed granular matter, and can be estimated thanks to the Darcy's law :

$$
\left.\frac{\Delta P}{L}\right|_{\text {Darcy }}=\frac{\eta_{0} v_{G}}{k},
$$

with the permeability $k$ of the granular packing.

In order to follow a generic approach encompassing a wide range of granular material properties, we decided to represent characteristic curves in a diagram with dimensionless variables along the axes. The typical pressure drop that we decided to use here is the Hagen-Poiseuille prediction for pure transporting fluid in eq. (1). A typical velocity of transported solid $v_{s}$ can be built out of the mass rate of transported granular matter $W$ :

$$
v_{s}=\frac{W}{\rho_{s} \phi_{c} \pi R^{2}},
$$

with the bulk density of the solid forming the grains $\rho_{s}$ adjusted by the filling fraction of the granular packing $\phi_{c}$, and the cross-section of the hydraulic line, $\pi R^{2}$ here.

Figure 6 shows in semi-logscale the characteristic curve for our study in dimensionless units $(\Delta P / L)^{*}=(\Delta P / L-$ $\left.\Delta P /\left.L\right|_{0}\right) /\left(\Delta P /\left.L\right|_{0}\right)$ and $v_{G}^{*}=v_{G} / v_{s}$. With this choice of variables the reduced pressure gradient $(\Delta P / L)^{*}$ shows a monotonic decreasing behavior. We used a fit of the form 


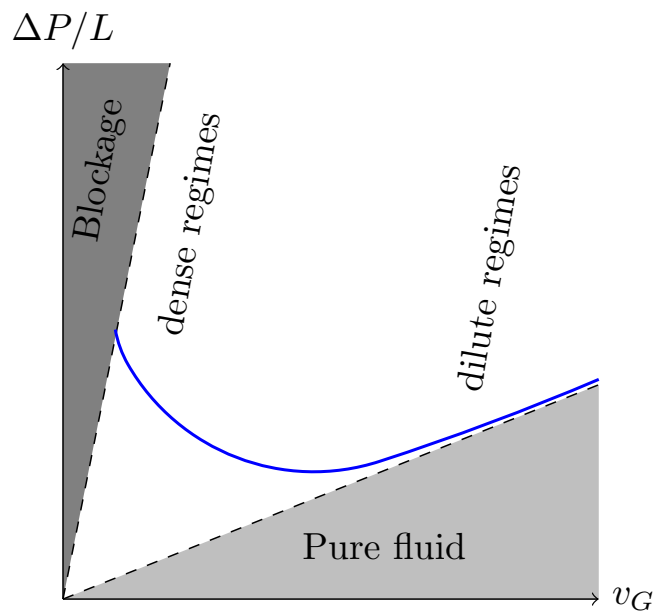

Figure 5. Schematical pressure drop $\Delta P / L v s$ carrying fluid velocity $v_{G}$ diagram used to represent specific curves (solid line). Limits due to pure fluid transport and obstruction are represented by the grayed and dark grayed areas.

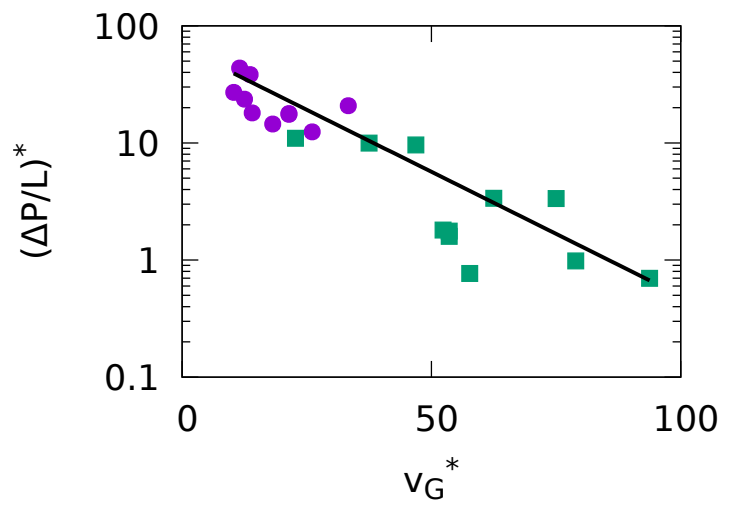

Figure 6. Characteristic curves diagram in dimensionless units $(\Delta P / L)^{*}$ vs $v_{G}^{*}$ in semilog scale. Symbols used follow the same definition as in fig. 4. The solid line is a fit for the experimental measurements.

$(\Delta P / L)^{*}=A \beta^{-v_{G}^{*}}$, with $A=65$ and $\beta=1.05$.

In this graph the viscous contribution to the pressure drop due to the carrying fluid flow is removed. The rescaled pressure drop $(\Delta P / L)^{*}$ tells about the magnitude of dissipative effects other than viscous dissipation within the fluid phase, most likely involving solid friction [8]. The relative magnitude of alternative dissipation processes are maximum for small rescaled carrying fluid velocities $v_{G}^{*}$. It is interesting to note that the transition in hydraulic transport regimes clearly seen in the experimental points Fig. 4 is no longer visible when the Hagen-Poiseuille viscous contribution is removed.

\section{Conclusion}

In this paper we studied the hydraulic transport of $100 \mu \mathrm{m}$ diameter glass beads through a horizontal millifluidic tube of inner radius $R=1 \mathrm{~mm}$ and length $L=59 \mathrm{~cm}$, with water flow rate $I_{0}$ imposed by a syringe pump. We observed two qualitatively different transport regimes (i) with formation and displacement of granular slugs at $I_{0} \in[1-4] \mathrm{mL} \cdot \mathrm{min}^{-1}$, (ii) with the movement of the whole granular sedimented bed at $I_{0} \in[5-20]$ $\mathrm{mL} \cdot \mathrm{min}^{-1}$. The crossover appears for $I_{0} \approx 4 \mathrm{~mL} \cdot \mathrm{min}^{-1}$. Measurements of the pressure drop imposed by the pump clearly show a maxima for imposed flow rate values close to the crossover between the two regimes. However the limited range of investigated flow rate values do not account for the increasing pressure drop expected for very large imposed flow rates.

The interpretation of the hydraulic transport system over the selected range of flow rates by means of dimensionless characteristic curves seems to show a global trend valid for both regimes. The pressure drop evolution as a power law of the superficial gas flow rate gives a reasonable predictive law for this system. Further work could be done to investigate the origin of this trend in terms of rheological behaviour.

The spatiotemporal diagrams obtained from image analysis done for hydraulic regimes with slugs formation seem to indicate an increase of the slugs speed with the imposed flow rate. A statistical analysis of the slugs sizes and speeds could give interesting informations about slug formation and travelling. This study at millifluidic scale recover typical transport regimes observed in hydraulic or even pneumatic systems. The generality of the observed phenomena would also be interesting to test for various granular materials, with different characteritics such as density, size or shape. Comparing the conclusions obtained at a millifluidic scale and at larger scales, closer to industrial scales, would be of great interest for a better understanding of granular matter behaviour and transport.

\section{References}

[1] P. Peyre, Metals 10, 459 (2020)

[2] A. Malagalage, W.K. Hiromi Ariyaratne, C. Ratnayake, M.C. Melaaen, Chem. Eng. Technol. 41, 1531 (2018)

[3] E. Guazzelli, O. Pouliquen, J. Fluid Mech. 852, P1 (2018)

[4] P. Doron, D. Barnea, Int. J. Multiphase Flow 22, 273 (1996)

[5] E. Rabinovitch, H. Kalman, Powder Technol. 207, 119 (2011)

[6] H. Kalman, A. Rawat, Chem. Eng. Sci. 211, 115256 (2020)

[7] M.M. Denn, J.F. Morris, Annu. Rev. Chem. Biomol. Eng. 5, 203 (2014)

[8] G. Dumazer, B. Sandnes, K.J. Måløy, E.G. Flekkøy, Phys. Rev. Fluids 5, 034309 (2020) 